Z Epileptol 2018 $\cdot 31: 41-47$ https://doi.org/10.1007/s10309-018-0175-0 Online publiziert: 24. Januar 2018 (c) Der/die Autor(en) 2018. Dieser Artikel ist eine Open-Access-Publikation.

CrossMark

\title{
Gerhard Luef
}

Abteilung für Neurologie, Medizinische Universität Innsbruck, Innsbruck, Österreich

\section{Hormon- und Sexualstörungen bei Epilepsie}

Weiteren haben Fortpflanzungshormone einen großen Einfluss auf die neuronale Erregbarkeit und als deren Folge auf epileptische Anfälle. Dies belegen sowohl klinische Beobachtungen als auch experimentelle Studien an Tieren.

\section{Reproduktive Dysfunktion bei Frauen mit Epilepsie}

Hospital- [2, 7, 9] und Community- [10] basierte Studien konnten zeigen, dass Störungen der Menstruation bei Frauen mit Epilepsie häufiger sind als in der Allgemeinbevölkerung.

Menstruationsstörungen können in Amenorrhö (keine Menses für 6 Monate), Oligomenorrhö (Zyklusintervalle $>32$ Tage), Polymenorrhö (Zyklusintervalle $<26$ Tage), abnormale Variationen der Zyklusintervalle ( $>4$ Tage) und Menometrorrhagie (starke Menses und Blutungen zwischen den Menses) kategorisiert werden [11].

Diese definierten Störungen können bei einem Drittel der Frauen mit Epilepsie auftreten, verglichen mit 12-14\% bei Frauen ohne Epilepsie $[6,11]$. Mehr als ein Drittel der Zyklen bei Frauen mit einer fokalen Epilepsie sind anovulatorisch, bei Kontrollen sind diese nur bei 8-10\% [11-13]. Es gibt widersprüchliche Beweise, dass anovulatorische Zyklen häufiger bei fokalen als bei generalisierten Epilepsien sind [12, 13].

Darüber hinaus finden sich gehäuft metabolische und endokrine Funktionsstörungen wie das PCO-Syndrom, Hyperandrogenämie, Gewichtszunahme und Insulinresistenz mit einem potenziellen Einfluss auf die ovarielle Funktion. Derartige pathologische Funktionsabweichungen treten bei Epilepsie sowohl als direkte Folge der Epilepsie als auch deren Therapie auf [2, 14-17]. Die Kenntnis der neurogonadalen Interaktionen zwischen gestörten zerebralen Erregungsimpulsen bei Frauen mit Epilepsie, dysfunktionellen Ovarien, Steroidwirkungen auf das Gehirn und dem variablen endokrinen und metabolischen Einflusspotenzial einer antiepileptischen Medikation ist die Grundlage eines rationalen Therapieansatzes, der nicht nur die antikonvulsive Wirkung der Medikation berücksichtigt. Im Folgenden werden die verschiedenen Ebenen möglicher Einflussfaktoren durch zusammenfassende klinische Beispiele zur ovariellen Insuffizienz bei Frauen mit Epilepsie (WWE) dargestellt und Therapieoptionen in Situationen mit und ohne Kinderwunsch erörtert.

\section{Hypothalamische Funktionsstörung}

Eine interiktale Erregungsstörung kann zu chronischen Störungen der Gonadotropinsekretion mit einer Veränderung des LH/FSH-Quotienten führen. Bei Frauen mit Epilepsie ohne AED-Therapie wird eine erhöhte LH-Pulsfrequenz beobachtet [18]. Zyklusstörungen traten allerdings bei den in dieser Studie untersuchten Patientinnen trotz der erhöhten LH-Pulse nicht gehäuft auf, obwohl diese insgesamt bei Frauen mit Epilepsie überrepräsentiert sind [19], und zwar unabhängig von einer AEDTherapie. Möglicherweise handelt es sich bei der hypothalamischen Dysregulation zunächst um subklinische Veränderungen, die einer Manifestation endokriner Störungen unter zusätzlichen Belastungsmomenten vorangehen und sie begünstigen. und von Östradiol sowie von Prolaktin konnte ebenso beobachtet werden. Des 
Neben den epileptischen Anfällen scheinen die interiktalen zentralnervösen Erregungsstörungen eine Dysbalance der Ausschüttung von regulärem LH und follikelstimulierendem Hormon (FSH) zu provozieren [7, 20]. Demzufolge propagieren gestörte ZNS-Erregungsabläufe, v. a. aus temporolimbischen Arealen, zu hypothalamischen Regulationszentren und interferieren mit der neuronalen Aktivität des hypothalamischen Pulsgenerators, der die GnRHSekretion steuert.

Möglicherweise variiert bei einer Temporallappenepilepsie (TLE) das resultierende, gestörte LH-Pulsatilitätsmuster abhängig von der Lateralität des epileptogenen Fokus [3, 21]. Ein rechtsseitiger Fokus führt häufiger zu einer LHSuppression und einem hypogonadotropen Hypogonadismus (hypothalamische Ovarialinsuffizienz). Dagegen tritt bei einem linksseitigen Fokus eher eine gesteigerte Gonadotropinausschüttung auf mit höheren LH-Werten und Anhebung der LH/FSH-Ratio. Dazu passend findet sich ein häufigeres Auftreten eines PCOS bei diesen Patientinnen [7, 22]. Gelingt die epilepsiechirurgische Ausschaltung des Fokus mit der gestörten neuronalen Aktivität, wird häufig eine Normalisierung der zuvor gestörten Zyklusfunktion erreicht [23].

\section{Antikonvulsive Medikamente und Steroidmetabolismus}

Enzyminduzierende antikonvulsive Medikamente führen durch Induktion der Isoenzyme CYP1, CYP2 und CYP3 des hepatischen Cytochrom-P-450-Enzymsystems zu einem beschleunigten Steroidhormonabbau v. a. durch das Isoenzym CYP3A4. Parallel dazu ist die SHBG-Synthese gesteigert. Dadurch wird die Bioverfügbarkeit einiger exogener und endogener Steroide (z.B. Testosteron, Östradiol, Gestagene) vermindert [8]. Die Wirksamkeit exogener Steroide kann dadurch eingeschränkt sein, was v. a. im Zusammenhang mit hormonellen Kontrazeptiva von Bedeutung ist [24]. Die Daten zum Einfluss von AED und im Besonderen der Valproinsäure (VPA) auf die Entstehung einer Hyperandrogenämie sind inhomogen und widersprüchlich. Ein durchgängiger Nachweis zur Entwicklung einer Hyperandrogenämie (HA) oder eines PCOS kann in Studien nicht gefunden werden. Unter einer VPA-Behandlung haben $6 \%$ [22] bis 27\% [5] der Patientinnen eine Hyperandrogenämie. Für andere AED (Carbamazepin, CBZ 17,1\%, Lamotrigin, LTG 4,5\%, Polytherapie $11,1 \%$ ohne AED $25 \%$ ) schwankt die Häufigkeit einer Hyperandrogenämie im gleichen Bereich. In einer der wenigen randomisierten klinischen Studien zu diesem Thema konnte im direkten Vergleich zwischen der Therapie mit VPA und Lamotrigin (LTG) kein signifikanter Unterschied in der Androgenproduktion beobachtet werden [25]. Eine weitere randomisierte Studie zum direkten Vergleich zwischen VPA und LTG bei jeweils über 100 Frauen ergab zwar eine signifikant erhöhte Androgenkonzentration im Serum der mit VPA behandelten Patientinnen, allerdings lagen die mittleren Androgenwerte bei beiden Gruppen im Normbereich [26].

Eine Gewichtszunahme kommt als Nebenwirkung einer Behandlung mit VPA bei $20-70 \%$ der Patienten vor [27]. Der exakte, zugrunde liegende pathophysiologische Mechanismus ist unklar. Möglicherweise sind Zustände einer Insulin- und Leptinresistenz ursächlich beteiligt, jedenfalls treten diese metabolischen Alterationen ebenfalls gehäuft auf. Als Folge einer solchen metabolischen Veränderung steigt möglicherweise das Risiko für die Entwicklung einer Hyperandrogenämie, zumindest bei einer Subgruppe von Patientinnen. Höhere Androgenwerte werden v. a. bei Patientinnen beobachtet, die während einer VPA-Behandlung mit Gewichtszunahme reagieren [28].

EIAED können durch die verminderte Bioverfügbarkeit der Androgene bei beschleunigter Metabolisierung und erhöhter SHBG-Bindung zu einem hormonellen „Kontrastierungseffekt “gegenüber nEIAED führen. Die Anwendung von EIAED mindert durch das induzierte enzymatische Metabolisierungsprofil die wirksame Androgenmenge in Relation zu nEIAED. Der Vergleich zwischen Therapiegruppen mit verschiedenen EIAED und behandlungsnaiven Pati- entinnen zeigte signifikant niedrigere Serumandrogenwerte in der Therapiegruppe gegenüber den nicht behandelten Kontrollpatientinnen [13, 29].

Keine Differenz der Serumandrogenkonzentration fand sich im Vergleich zwischen Patientinnen in einer VPABehandlungsgruppe und nicht medikamentös behandelten Patientinnen [30, 31]. Ein weiterer Hinweis auf einen möglichen „Kontrastierungseffekt" ergibt sich aus der Verlaufsbeobachtung nach Absetzen der AED. Im Serum gemessene Androgenkonzentrationen steigen nach Beendigung einer Behandlung mit CBZ wieder an. Dieser Effekt konnte für VPA nicht gezeigt werden [32].

Lamotrigin (LTG) wird durch therapeutische Östrogen- und Gestagendosierungen (z. B. in hormonellen Kontrazeptiva) selbst schneller abgebaut [33], was als eine Folge der gesteigerten Aktivität der UDP-Glucuronosyltransferase 1A4 angesehen wird [34]. Eine Hormonbehandlung zur Kontrazeption oder Hormonersatztherapie erfordert eine Dosissteigerung von LTG um etwa 30-50\% innerhalb einer Woche nach Beginn der Hormongabe.

\section{Polyzystisches Ovarialsyndrom (PCOS)}

Die häufigste reproduktive endokrine Störung bei Frauen mit Epilepsie als auch in der Allgemeinbevölkerung ist das polyzystische Ovarialsyndrom (PCOS) $[2,15,17]$.

Das PCOS ist durch 3 Hauptsymptome charakterisiert: Zyklusstörungen mit Oligo- oder Anovulation (AO), klinische oder laborchemische Hyperandrogenämie (HA) und spezifische polyfollikuläre Alteration der Ovarmorphologie (PCO). Nach den RotterdamKriterien müssen 2 der 3 Symptome (AO, HA, PCO) in variabler Kombination vorliegen. Andere Ursachen einer Hyperandrogenämie müssen ausgeschlossen sein. Ein so definiertes PCOS hat in unseren Breiten eine Prävalenz von ca. $12 \%$ und stellt somit die häufigste Ursache einer Ovarialinsuffizienz bei Frauen in der reproduktiven Lebensphase dar. Nach den NIH-Kriterien (AO, HA) liegt die Prävalenz bei 6-8\%. 
Neben den genannten Diagnosekriterien liegen gehäuft typische endokrine und metabolische Abweichungen im Sinne einer Insulinresistenz und eines metabolischen Syndroms vor [35].

Frauen mit Epilepsie haben ein erhöhtes Risiko, ein PCOS zu entwickeln. Die Angaben zum Einfluss, den AED allgemein oder Einzelsubstanzen wie etwa Valproinsäure (VPA) haben, schwanken in vergleichbarer Breite wie die oben genannten Prävalenzzahlen. Eine Vielzahl von Publikationen verweist auf ein deutlich erhöhtes Risiko eines PCOS unter einer VPA-Behandlung, seit die finnische Arbeitsgruppe um Isojärvi 1993 diesen Zusammenhang in einer Querschnittstudie erstmalig berichtete [5]. Allerdings zeigt die umfangreiche vergleichende Auswertung diesbezüglicher Publikationen anderer Autoren keineswegs eine so eindeutige Zuordnung zwischen Medikation und Symptomatik, sodass diese Frage nach wie vor ungeklärt ist [16].

Zur Prävalenz und Ätiologie des PCOS bei Frauen mit Epilepsie liegen widersprüchliche Angaben vor. Die Angaben schwanken allerdings erheblich zwischen 10,5\% [36] und 62,5\% [14]. Zusammenfassend kann man sagen, dass das PCOS bei Frauen mit Epilepsie häufiger vorkommt als ohne Epilepsie. Die ätiologische Gewichtung des morbogenen Einflusses sowie der direkten und der indirekten Einflüsse verschiedener einzelner AED auf die Entwicklung eines PCOS werden immer noch höchst kontrovers diskutiert. Die hierzu publizierte Literatur ist großenteils durch mangelhaftes Studiendesign (zu geringe Fallzahlen, unzureichende, teilweise willkürliche Definition des PCOS, Fokussierung auf das Auftreten einzelner Aspekte des PCOS) ungeeignet, eine evidenzbasierte Aussage zu treffen. Im Speziellen kann eine Sonderstellung von Valproinsäure gegenüber anderen AED für die Genese eines PCOS nicht überzeugend belegt werden. Das Gesamtbild bleibt fragmentarisch. Besondere Aufmerksamkeit muss bei der Behandlung einer Patientin mit Epilepsie auf die Entwicklung von Symptomen wie $\mathrm{Zy}$ klusstörungen, Gewichtszunahme und klinische Zeichen der Hyperandrogenämie gelegt werden. Diese können eine

Z Epileptol 2018 · 31:41-47 https://doi.org/10.1007/s10309-018-0175-0

(c) Der/die Autor(en) 2018. Dieser Artikel ist eine Open-Access-Publikation.

G. Luef

\section{Hormon- und Sexualstörungen bei Epilepsie}

\section{Zusammenfassung}

Die Behandlung von Menschen mit Epilepsie stellt behandelnde Ärzte vor komplexe Anforderungen. Epilepsien, Antikonvulsiva (AEDs) und das reproduktive System haben komplexe Interaktionen. Störungen der Reproduktion sind relativ häufig bei Menschen mit Epilepsie. Sie sind generell assoziiert mit und können die Folge von reproduktiven endokrinen Störungen sein. Es ist nicht dasselbe, eine Frau oder ein Mann mit Epilepsie zu sein. Eine gute Betreuung epilepsiekranker Frauen und Männer zielt längst nicht mehr nur auf Anfallsfreiheit ab, sondern auch auf eine nebenwirkungsarme Therapie, die alle Lebensbereiche so wenig wie möglich einschränkt. Einerseits können hormonelle Veränderungen bei Frauen mit Epilepsie während des menstrualen Zyklus das Auftreten von epileptischen Anfällen begünstigen, andererseits können epileptische
Anfälle zu Veränderungen der zirkulierenden Hormone (Gonadotropine, Prolaktin, Sexualsteroide) führen. Es bestehen multidirektionale neurogonadale Interaktionen zwischen den Impulsen der zentralnervösen Erregungsstörung im Anfallsgeschehen sowie interiktal mit resultierender Dysbalance der hypothalamisch-hypophysären Funktion, dem neuroaktiven Potenzial der gonadalen Steroide und der endokrinen Modulation durch die antiepileptische Medikation (AED). Bei Männern kann dies nach einzelnen Studien zu Verlust von Libido, erektiler Dysfunktion, Veränderungen der Gonaden und der Spermienqualität führen.

Schlüsselwörter

Epilepsie · Hormonstörungen · Fertilität . PCOS · Hypogonadismus · Antikonvulsiva

\section{Hormonal and sexual disorders in epilepsy}

\section{Abstract}

Disorders of reproduction are unusually common among people with epilepsy. They are generally associated with and may be the consequence of reproductive endocrine disorders. Epilepsy itself and epileptic seizures have been implicated in the pathophysiology. Fertility is lower in both men and women with epilepsy than in the general population. Temporolimbic dysfunction in epilepsy may disrupt normal neuroendocrine regulation and promote the development of reproductive endocrine disorders. The particular nature of the dysregulation may relate to the laterality and focality of the epilepsy and some hormonal changes may develop in close temporal relation to the occurrence of epileptiform discharges. In women, reproductive endocrine disorders include polycystic ovary syndrome, hypothalamic amenorrhea, functional hyperprolactinemia, and premature menopause. The use of valproic acid (VPA) is associated with a frequent occurrence of reproductive endocrine disorders characterized by polycystic changes in the ovaries, high serum testosterone concen- trations (hyperandrogenism) and menstrual disorders. In men, hypogonadism may be hypogonadotropic, hypergonadotropic and changes of the quality of the spermatozoa have been observed. The endocrinal effects of new antiepileptic drugs (AEDs) have not been widely studied; however, it seems that they may be an alternative if reproductive endocrine problems emerge during treatment with older antiepileptic drugs. The use of the liver enzyme-inducing AEDs phenobarbital, phenytoin and carbamazepine increases serum sex hormone-binding globulin (SHBG) concentrations in both men and women with epilepsy. Over time the increase in serum SHBG levels leads to diminished bioactivity of testosterone and estradiol, which may result in diminished potency in men and menstrual disorders in some women and thus, reduced fertility.

Keywords

Epilepsy - Reproductive disorders - Fertility . PCOS · Hypogonadism · Antiepileptic drugs 
beginnende Endokrinopathie oder metabolische Störung anzeigen. Frauen mit Epilepsie haben ein erhöhtes Risiko zur Entwicklung von Übergewicht, Insulinresistenz, verminderter hepatischer Insulinclearance, Leptinresistenz und Dyslipidämie. Dieser Sachverhalt ist der verwendeten antiepileptischen Medikation nicht immer eindeutig zuzuordnen, aber möglicherweise unter VPA-Therapie besonders akzentuiert [35].

\section{Reproduktive Dysfunktion bei Männern mit Epilepsie}

Verminderte Libido oder Potenz tritt beinahe bei $20 \%$ der Männer mit Epilepsie in strukturierten und unstrukturierten Fragebögen-Studien auf [4, 37]. Ältere Studien mit strukturierten oder unstrukturierten Interviews fanden diese noch häufiger bei 38-71\% [3, 4, 6, 38-40].

Abnormale Spermienanalysen, mit verminderter Spermienzahl, abnormaler Morphologie oder verminderter Motilität der Spermien, wurde bei bis zu 90\% von Männern mit Epilepsie berichtet [38, 41].

\section{Hypogonadismus}

Hypogonadismus bedeutet niedrige Serumtestosteronspiegel und/oder verminderte oder abnormale Spermienproduktion [6]. Es zeigen sich vermindertes sexuelles Interesse, verminderte Potenz, Fertilität, Energie, Stimmung, Knochen und Muskelmasse und sekundäre sexuelle Charakteristika. Physikalische Zeichen beinhalten einen Verlust von männlichen Merkmalen, Gynäkomastie und Atrophie der Gonaden.

Testosteron besteht aus 3 Hauptformen: 1) streng an Sexualhormonbindungsglobulin gebunden (SHBG, 45-50\%), locker an Albumin gebunden (50-55\%) und 3) ungebunden (1-2\%) [6]. Das Albumin-gebundene und freie Testosteron sind für Gewebe verfügbar und daher von klinischer Relevanz. Untersuchungen des bioverfügbaren Testosterons (BAT) zeigen, dass ein Hypogonadismus in etwa bei einem Drittel der Männern mit Temporallappenepilepsie auftreten kann [4, 37]. BAT vermindert sich altersabhängig bei Männer mit Epi- lepsie rascher als bei Kontrollen [4, 37]. In einer Kohorte von Männern mit fokaler Epilepsie fanden Herzog et al., dass das BAT deutlich unter den normalen Kontrollspiegel zurückfiel: um $11 \%$ bei Männer zwischen 20 und 30 Jahren, um $27 \%$ zwischen 30 und 40 Jahren und um $89 \%$ zwischen 40 und 50 Jahren [4]. Einige [4, 42, 43], aber nicht alle [44] Studien haben eine signifikante Beziehung zwischen vermindertem SerumBAT und sexueller Dysfunktion gezeigt. Männer mit Epilepsie zeigen bei niedrignormalen BAT-Spiegeln ein Vorhandensein einer sexuellen Dysfunktion, welche bei Männern der Allgemeinbevölkerung sich nicht klinisch manifestiert $[3,4,6]$. Dies könnte ein Argument gegen die Bedeutung der BAT-Spiegel sein. Andererseits werden höhere BAT-Spiegel bei verändertem Gehirnsubstrat im Rahmen einer temporolimbischen Epilepsie für eine normale Sexualfunktion benötigt [37].

\section{Pathophysiologie von reproduktiven endokrinen Störungen bei Männern mit Epilepsie}

Ätiologisch können sowohl für einen Hypogonadismus als auch für reproduktive und sexuelle Dysfunktionen bei Männern mit Epilepsie eine Vielzahl von Ursachen in Betracht gezogen werden. Das wären sowohl psychosozialer Stress, AEDs als auch Epilepsien selbst [6].

AEDs können die Sexualfunktion beim Mann teilweise erheblich beeinflussen. Es kann zum Hypogonadismus kommen, zur Störung der endokrinen und/oder exokrinen Hodenfunktion.

Das bedeutet, es kann zu einer Verminderung der Testosteronausschüttung kommen mit typischen klinischen Beschwerden.

Bereits in den 1850er-Jahren, als Brom zur Behandlung von Epilepsien eingeführt wurde, sah man, dass diese Salze zu Impotenz führen können. Später zeigte eine randomisierte Medikamentenstudie nach einem Therapiebeginn von Carbamazepin (CBZ), Phenytoin (PHT), Phenobarbital oder Primidon in Monotherapie bei 11-22\% der Männer die Entwicklung von Impotenz oder verminderter Li- bido. Endokrine Daten wurden damals noch nicht erhoben.

Erste Studien, die von endokrinen Störungen bei Männern mit Antikonvulsivatherapie berichten, wurden in den 1970er- und 1980er-Jahren veröffentlicht. Es wurde ein Anstieg von einem SHBG-Serumspiegel und Abfall des freien (bioaktiven) Testosterons beschrieben. Begleitend dazu wurden verminderte Sexualfunktionen gefunden. SHBG wird in der Leber synthetisiert und bindet und reguliert die Bioaktivität von Testosteron und Östradiol.

In den meisten anfänglichen Studien waren die Patienten jedoch mit einer Polytherapie, welche alle Enzym induzierende Medikamente enthielten, behandelt, was die Beurteilbarkeit dieser Studienergebnisse einschränkt.

Psychosozialer Stress kann bei Hypogonadismus in Verbindung mit einer Epilepsie eine wichtige Rolle spielen [45-50]. Aus der neuroendokrinen Perspektive involviert eine Stressantwort die Aktivierung der hypothalamisch-hypophysären (HPA) Achse [6, 46-50]. Cortisolspiegel sind bei Menschen mit Epilepsie höher als bei Kontrollen und ähnlich wie bei Menschen mit einer Depression [47]. Anders als bei einer Depression sind bei Epilepsien die täglichen Schwankungen jedoch oft nicht vorhanden [47]. Faktoren, die die Aktivität der HPA-Achse erhöhen, stören sowohl die reproduktive endokrine Sekretion als auch die reproduktive Funktion [6, 46-50] und können zu einer Anfallsexazerbation beitragen [6].

Stress erhöht die Freisetzung von Proopiomelanocortin (POMC), ein Protein und Prohormon der corticotropen Zellen der Adenohypophyse und des Hypothalamus, das unter anderem zur Bildung von ACTH und Endorphin gespalten wird [50]. Beide hemmen die Gonadotropinsekretion und die reproduktive Funktion [46-50]. ACTH erhöht die Kortisolsekretion; Endorphine erhöhen die Dehydroepiandrosteron (DHEA)-Produktion. Diese beiden Steroide haben eine GABA-negative allosterisch modulierende Eigenschaft, welche die Anfallsschwelle erniedrigen und Angst erhöhen können [6, 37, 51].

Enzym induzierende AEDs können die gonadale Testosteronsynthese direkt 
unterdrücken, die Testosteronbindung durch Induktion der Sexhormonbindungsglobulin (SHBG)-Synthese erhöhen und Serumöstradiolspiegel in absoluter oder relativer Hinsicht erhöhen [4, $37,52]$. Obwohl es nur etwa $1 \%$ der gesamten reproduktiven Steroide beträgt, macht Östradiol die Hälfte des negativen Feedbacks der hypothalamischenhypophysären Achse aus [6,37]. Daher kann ein geringer Anstieg des Östradiolspiegels, vermutlich durch AED induzierte Erhöhung der Aromataseaktivität verursacht, einen disproportionalen großen negativen Feedbackeffekt auf die Gonadotropinproduktion haben, was zu Hypogonadismus beiträgt. In einem Vergleich der sexuell/reproduktiven Funktion und reproduktiven Hormonspiegel bei 85 Männern mit Epilepsie, die unterschiedliche AEDs einnahmen (25 Carbamazepin [CBZ], 25 Phenytoin [PHT], 25 Lamotrigin [LTG] und 10 unbehandelt in den mindestens letzten 6 Monaten [keine AED]) und 25 Kontrollen fanden Herzog et al. [4], dass sexuelle Funktionscores („S-scores"), Hormonspiegel (bioaktives Testosteron, Östradiol), Hormonverhältnisse (bioaktives Testosteron/bioaktives Östradiol) und gonadale Effizienz (bioaktives Testosteron/luteinisierendes Hormon) signifikant höher als bei den Kontrollen und in der LTG-behandelten Gruppe als in der CBZ- und PHT-behandelten Gruppe waren. Das Sexhormonbindungsglobulin war signifikant höher in der CBZund PHT-Gruppe als in allen anderen Gruppen. „S-scores“ waren unter der Kontrollreichweite bei $20 \%$ der Männer mit Epilepsie, inkludiert von $32 \% \mathrm{mit}$ CBZ, $24 \%$ mit PHT, $20 \%$ ohne AEDs und $4 \%$ unter LTG. Bioaktives Testosteron war unter der Kontrollreichweite bei $28,2 \%$, inkludiert von $48 \%$ mit CBZ, $28 \%$ mit PHT, $20 \%$ ohne AEDs und $12 \%$ unter LTG. Bei Männern mit Epilepsie mit niedrigen „S-scores“ hatten $70,6 \%$ bioaktive Testosteronspiegel unter der Kontrollreichweite, verglichen mit 17,6\% Männern mit normalen „Sscores“. Bei Männern mit Epilepsie und abnormal niedrigem bioaktivem Testosteron hatten 50,0\% niedrige „S-scores“; bei Männern mit normalen bioaktiven Testosteron hatten $8,2 \%$ niedrige „S- scores“. Die bioaktive Testosteronverminderung gemessen am Alter war bei Männern mit Epilepsie höher als unter Kontrollen und sichtbar höher in den CBZ- und PHT-Gruppen als in den LTG und unbehandelten Gruppen. Insgesamt waren bei Männern mit Epilepsie, die LTG einnahmen, die Sexualfunktionen, bioverfügbare Testosteronspiegel und gonadale Effizienz vergleichbar mit Kontrollen und unbehandelten Werten und signifikant höher als mit Carbamazepinoder Phenytoin-Behandlung.

Das temporolimbische System ist eine der häufigsten Ursprungsstellen von Epilepsien im Erwachsenenalter. Es hat auch integrale Rollen in der reproduktiven endokrinen Regulation und dem Feedback als auch bei sexuellen und reproduktiven Funktionen [3, 6, 37]. Es erklärt sich daher, dass die Entwicklung von epileptiformen Entladungen in den mesialen Temporallappenstrukturen die hypothalamische Regulation der Hypophysensekretion unterbricht und daher die gonadale und reproduktive Funktion ändert [3, 6, 37]. Experimentelle Tierversuche haben gezeigt, dass fokale limbische, aber auch generalisierte Anfälle normale gonadale Strukturen, Physiologie und Serumandrogenspiegel in männlichen Ratten unterbrechen [53] und dies die Induktion von Anfällen in der Amygdala und nicht im Motorkortex Hyposexualität bei männlichen Katzen verursacht [54]. Klinische Studien legen nahe, dass eine temporolimbische Epilepsie mit verändertem Gonadotropin-Response auf eine GnRH-Infusion unabhängig von AED-Gebrauch assoziiert ist [55]. Epileptiforme Entladungen bei Männern sind von einer akuten Prolaktinerhöhung begleitet [56] und können auch von akuten Veränderungen der LH-Sekretion begleitet sein [6]. Quigg et al. [57] haben gefunden, dass die LH-Pulsfrequenz und die mittlere Konzentration interiktal niedriger und die Pulsamplituden höher als bei Kontrollen sind. Postiktal haben sie keine Veränderung der mittleren Pulsfrequenz gefunden, aber sie fanden eine signifikante Veränderung in der Regulation des Vorkommens von LH-Pulsen. Klinisch kommt es bei Männern mit rechts lateralisierender temporolimbischer Epilepsie zu häufigerem Auftreten sexueller Dysfunktionen als mit links lateralisierender $[3,37,58]$. Es gibt noch aus Tierexperimenten zusätzliche Evidenz, dass die linke und die rechte Seite des Hypothalamus einen unterschiedlichen Effekt auf die Sexualfunktion ausüben [59]. Die Testosteronspiegel können bei Männern mit einem temporalen Fokus niedriger verglichen mit Männern mit extratemporalen Foci sein [60]. Erfolgreiche Temporolobektomie bei hypogonadalen Männern mit therapieresistenten Anfällen wurde mit einer Normalisierung der Testosteronspiegel assoziiert [61] und eine Verbesserung des sexuellen Interesses und der Sexualfunktion festgestellt [62]. Eine bemerkenswert hohe Frequenz an abnormalen Befunden von Samenanalysen wurde sowohl bei unbehandelten als auch bei behandelten Männern mit Epilepsie berichtet [4, 63].

Veränderungen der reproduktiven Steroidkonzentrationen können sich auf die Anfallssituation als auch auf die reproduktive Funktion auswirken. Während Östrogen prokonvulsiv und Progesteron antikonvulsiv ist, scheint der Effekt von Testosteron in den meisten Tiermodellen mit fokalen Epilepsien unterschiedlich zu sein. Dies kann einerseits durch den abgeschlossenen Metabolismus zu Östradiol mithilfe der Aromatase sein [64], oder es kann auch durch die Reduktase zu Dihydrotestosteron metabolisiert werden und weiter zu Androstanediol, einem potenten GABAergem Steroid mit Antianfallseigenschaften [6, 65].

\section{Fazit für die Praxis}

\section{- Störungen der Fortpflanzungsfähig- keit sind bei Frauen und Männern mit Epilepsie häufiger als in der Normalbevölkerung. \\ - Störungen der Hypothalamus-Hy- pophysen-Achse (HPA) sind durch Temporallappenanfälle und durch Einflüsse der AEDs auf den Steroid- hormonstoffwechsel möglich. \\ - An endokrinen Syndromen findet man das polyzystische Ovarial- syndrom (PCOS), hypothalamische Amenorrhöen (HA) bei Frauen und erektile Dysfunktionen verbunden}


mit Libidoverlust und pathologische Veränderungen der Spermienqualität bei Männern mit Epilepsie.

- Enzyminduzierende Antiepileptika zeigten einen signifikanten Abfall von LH und Östradiol sowie einen Anstieg von SHBG und Prolaktin. Die durch den SHBG-Anstieg vermehrte Proteinbindung senkt die Serumkonzentrationen der frei wirksamen Sexualsteroide bei Männern und Frauen.

\section{Korrespondenzadresse}

Ao. Univ. Prof. Dr. G. Luef

Abteilung für Neurologie, Medizinische Universität Innsbruck

Anichstr. 35, 6020 Innsbruck, Österreich gerhard.luef@i-med.ac.at

Funding. Open access funding provided by University of Innsbruck and Medical University of Innsbruck.

\section{Einhaltung ethischer Richtlinien}

Interessenkonflikt. G. Luef gibt an, dass kein Interessenkonflikt besteht.

Dieser Beitrag beinhaltet keine vom Autor durchgeführten Studien an Menschen oder Tieren.

Open Access. Dieser Artikel wird unter der Creative Commons Namensnennung 4.0 International Lizenz (http://creativecommons.org/licenses/by/4.0/deed. de) veröffentlicht, welche die Nutzung, Vervielfältigung, Bearbeitung, Verbreitung und Wiedergabe in jeglichem Medium und Format erlaubt, sofern Sie den/die ursprünglichen Autor(en) und die Quelle ordnungsgemäß nennen, einen Linkzur Creative Commons Lizenz beifügen und angeben, ob Änderungen vorgenommen wurden.

\section{Literatur}

1. Herzog AG (1989) A hypothesis to integrate partial seizures of temporal lobe origin and reproductive disorders. Epilepsy Res 3:151-159

2. Herzog AG, Seibel MM, Schomer DL et al (1986) Reproductive endocrine disorders in women with partial seizures of temporal lobe origin. Arch Neurol 43:341-346

3. Herzog AG, Seibel MM, Schomer DL et al (1986) Reproductive endocrine disorders in men with partial seizures of temporal lobe origin. Arch Neurol 43:347-350

4. Herzog AG, Drislane FW, Schomer DL et al (2005) Differential effects of antiepileptic drugs on sexual function and reproductive hormones in men with epilepsy. Neurology 65:1016-1020

5. Isojarvi Jl, Laatkainen TJ, Pakarinen AJ et al (1993) Polycystic ovaries and hyperandrogenism in women taking valproate for epilepsy. N Engl J Med329:1383-1388

6. Herzog AG (2002) Reproductive endocrine regulation in men with epilepsy: effects on reproductive function and neuronal excitability. Ann Neurol 51:539-542

7. Herzog AG, Coleman AE, Jacobs AR et al (2003) Interictal EEG discharges, reproductive hormones and menstrual disorders in epilepsy. Ann Neurol 54:625-637

8. Anderson GD (2004) Pharmacogenetics and enzymeinduction/inhibition properties of antiepileptic drugs. Neurology 63(10Suppl4):S3-S8

9. Hamed SA, Hamed EA, Shokry M, Omar H, Abdellah MM (2007) The reproductive conditions and lipid profile in females with epilepsy. Acta Neurol Scand 115:12-22

10. Svalheim S, Taubøll E, Bjørnenak T et al (2003) Do women with epilepsy have increased frequency of menstrual disturbances? Seizure 12:529-533

11. Herzog AG, Friedman MN (2002) Menstrual cycle interval and ovulation in women with localizationrelated epilepsy. Neurology 57:2133-2135

12. Morrell MJ, Giudice L, Flynn KL et al (2002) Predictors of ovulatory failure in women with epilepsy. Ann Neurol 52:704-711

13. Lofgren $E$, Mikkonen $K$, Tolonen U, Pakarinen A, Koivunen R, Myllyla VV, Tapanainen JS, Isojarvi Jl (2007) Reproductive endocrine function in women with epilepsy: the role of epilepsy type and medication. Epilepsy Behav 10:77-83

14. Prabhakar S, Sahota P, Kharbanda PS, Siali R, Jain V, Lal V et al (2007) Sodium valproate, hyperandrogenism and altered ovarian function in Indian women with epilepsy: a prospective study. Epilepsia 48(7):1371-1377

15. Bilo L, Meo R, Nappi C et al (1988) Reproductive endocrine disorders in women with primary generalized epilepsy. Epilepsia 29:612-619

16. Bilo L, Meo R (2008) Polycystic ovary syndrome in women using valproate: a review. Gynecol Endocrinol 24(10):562-570

17. Herzog AG, Schachter SC (2001) On the association between valproateand polycysticovary syndrome. Epilepsia 42:311-315

18. Bilo L, Meo R, Valentino R, Buscaino GA, Striano S, Nappi C (1991) Abnormal pattern of luteinizing hormone pulsatility in women with epilepsy. Ferti Steril 55(4):705-711

19. Bilo L, Meo R, Valentino R, Di Carlo C, Striano S, Nappi C (2001) Characterization of reproductive endocrine disorders in women with epilepsy. J Clin Endocrinol Metab 86(7):2950-2956

20. Meo R, Bilo L, Nappi C, Tommaselli AP, Valentino $R$, Nocerino $C$ et al (1993) Derangement of the hypothalamic $\mathrm{GnRH}$ pulse generator in women with epilepsy. Seizure 2(3):241-252

21. Drislane FW, Coleman AE, Schomer DL, Ives J, Levesque LA, Seibel MM et al (1994) Altered pulsatile secretion of luteinizing hormone in women with epilepsy. Neurology 44(2):306-310

22. Quigg M, Smithson SD, Fowler KM, Sursal T, Herzog AG (2009) Laterality and location influence catamenial seizure expression in women with partial epilepsy. Neurology 73(3):223-227

23. Bauer J, Stoffel-Wagner B, Flügel D, Kluge M, Elge CE (2000) The impact of epilepsy surgery on sex hormones and the menstrual cycle in female patients. Seizure 9(6):389-393

24. Deichmann S, Luef G (2013) Carbamazepine coadministration with an oral contraceptive. Epilepsia 54(5):953

25. Stephen LJ, Sills GJ, Leach JP, Butler E, Parker P, Hitiris $N$ et al (2007) Sodium valproate versus lamotrigine: a randomised comparison of efficacy, tolerability and effects on circulating androgenic hormones in newly diagnosed epilepsy. Epilepsy Res 75(2-3):122-129

26. Morrell MJ, Isojärvi J, Taylor AE, Dam M, Ayala R, Gomez Getal (2003) Higherandrogens and weight gain with valproate compared with lamotrigine for epilepsy. Epilepsy Res 54(2-3):189-199

27. Verrotti A, D'Egidio C, Mohn A, Coppola G, Chiarelli $F$ (2011) Weight gain following treatment with valproic acid: pathogenetic mechanisms and clinical implications. Obes Rev 12(5):e32-e43

28. Herzog AG (2008) Disorders of reproduction in patients with epilepsy: primary neurological mechanisms. Seizure 17(2):101-110

29. Sahota P, Prabhakar S, Kharbanda PS, Bhansali A, Jain V, Das CP etal (2008) Seizure type, antiepileptic drugs, and reproductive endocrine dysfunction in Indian women with epilepsy: a cross-sectional study. Epilepsia 49(12):2069-2077

30. Murialdo G, Galimberti C (1998) Effects of valproate, phenobarbital, and carbamazepine on sex steroid setup in women with epilepsy. Clin Neuropharmacol 21:52-58

31. Betts T, Yarrow H, Dutton N, Greenhill L, Rolfe T (2003) A study of anticonvulsant medication on ovarian function in a group of women with epilepsy who have only ever taken one anticonvulsant compared with a group of women without epilepsy. Seizure 12(6):323-329

32. Lossius MI, Taubøll E, Mowinckel P, Mørkrid L, Gjerstad L (2007) Reversible effects of antiepileptic drugs on reproductive endocrine function in men and women with epilepsy - a prospective randomized double-blind withdrawal study. Epilepsy Behav 48(10):1875-1882

33. Christensen J, Petrenaite V, Atterman J, Sidenius P, Ohman I, Tomson T et al (2007) Oral contraceptives induce lamotrigine metabolism: evidence from a double-blind, placebo-controlled trial. Epilepsia 48(3):484-489

34. Chen H, Yang K, Choi S, Fischer JH, Jeong H (2009) Up-regulation of UDP-glucuronosyltransferase (UGT) 1 A4 by 17beta-estradiol: a potential mechanism of increased lamotrigine elimination in pregnancy. Drug MetabDispos 37(9):1841-1847

35. Rösing B, Wild L (2015) Neurogonadale Interaktionen bei Frauen mit Epilepsie. Z Epileptol 28(4):271-278

36. Bauer J, Jarre A, Klingmüller D, Elger CE (2000) Polycystic ovary syndrome in patients with focal epilepsy: a study in 93 women. Epilepsy Res 41(2):163-167

37. Herzog AG (2015) Disorders of reproduction in patients with epilepsy. ZEpileptol 28(4):271-278

38. Taneja N, Kucheria K, Jain S, Maheshwari MC (1994) Effect of phenytoin on semen. Epilepsia 35:136-140

39. Stoffel-Wagner B, Bauer J, Flugel D et al (1998) Serum sex hormones are altered in patients with chronic temporal lobe epilepsy receiving anticonvulsantmedication. Epilepsia 39:1164-1173

40. MacPhee JA, Larkin JG, Butler E, Graham HB, Brodie MJ (1988) Circulating hormones and pituitary responsiveness in young epileptic men receiving long-term antiepileptic medication. Epilepsia 29:468-475

41. Christiansen P, Deigaard J, Lund M (1975) Potens, fertilitet of konshormonudskillelse hos yngre manglige epilepsilidend. Ugeskr Laeger 137:2402

42. Fenwick PBC, Mercer C, Grant R et al (1986) Nocturnal penile tumescence and serum testosterone levels. Arch Sex Behav 15:13-21 
43. Toone BK, Wheeler M, Nanjee $M$ et al (1983) Sex hormones, sexual activity and plasma anticonvulsant levels in male epileptics. J Neurol Neurosurg Psychiatr 46:824-826

44. Duncan S, Blacklaw J, Beastall GH, Brodie MJ (1999) Antiepileptic drug therapy and sexual function in men with epilepsy. Epilepsia 40:197-204

45. Taylor DC (1969) Sexual behavior and temporal lobe epilepsy. Arch Neurol 21:510-516

46. Sapolsky RM (1985) Stress-induced suppression of testicular function in the wild baboon: role of glucocorticoids. Endocrinology 116:2273-2278

47. Gallagher BB, Murvin A, Flanigin HF et al (1984) Pituitary and adrenal function in epileptic patients. Epilepsia 25:683-689

48. Clarke D, Fearon U, Cunningham SK, McKenna TJ (1996) The steroidogenic effects of b-endorphin and joining peptide: a potential role in the modulation of adrenal androgen production. JEndocrinol 151:301-307

49. van Goozen SHM, van den Ban E, Matthys W et al (2000) Increased adrenal androgen functioning in children with oppositional defiant disorder: a comparison with psychiatric and normal controls. JAm Acad Child Adolesc Psychiatry 39:1446-1451

50. Sapolsky RM, Krey LC (1988) Stress-induced suppression of luteinizing hormone concentrations in wild baboons: role of opiates. J Clin Endocrinol Metab 66:722-726

51. Jacobs AR, Edelheit PB, Coleman AE, Herzog AG (1999) Late onset congenital adrenal hyperplasia: a treatable cause of anxiety. Biol Psychiatry 46:856-859

52. Murialdo G, Galimberti CA, Fonzi S et al (1995) Sex hormones and pituitary function in male epileptic patients with altered or normal sexuality. Epilepsia 36:358-363

53. Edwards HE, Mclntyre Burnham W, MacLusky NJ (1999) Partial and generalized seizures affect reproductive physiology differentially in the male rat. Epilepsia 40(11):1490-1498

54. Feeney DM, Gullotta FP, Gilmore W (1998) Hyposexuality produced by temporal lobeepilepsy in the cat. Epilepsia 39:140-149

55. Herzog AG, Russell V, Vaitukaitis JL, Geschwind N (1982) Neuroendocrine dysfunction in temporal lobe epilepsy. Arch Neurol 39:133-135

56. Sperling MR, Pritchard PB, Engel J, Daniel C, Sagel J (1986) Prolactin in partial epilepsy: an indicator of limbic seizures. Ann Neurol 20:716-722

57. Quigg M, Kiely JM, Shneker B et al (2002) Interictal and postictal alterations of pulsatile secretion of luteinizing hormone in temporal lobe epilepsy in men. Ann Neurol 51:559-566

58. Bear DM, Fedio P (1977) Quantitative analysis of interictal behavior in temporal lobe epilepsy. Arch Neurol 34:454-467

59. Nordeen EJ, Yahr P (1982) Hemispheric asymmetries in the behavioral and hormonal effects of sexually differentiating mammalian brain. Science 218:391-393

60. Bauer J, Blumenthal S, Reuber M, Stoffel-Wagner B (2004) Epilepsy syndrome, focus location, and treatment choice affect testicular function in men with epilepsy. Neurology 62:243-246

61. Bauer J, Stoffel-Wagner B, Flugel D, Kluge M, Schramm J, Bidlingmaier F, Elger CE (2000) Serum androgens return to normal after temporal lobe epilepsy surgery in men. Neurology 55:820-824

62. Blumer D, Walker AE (1967) Sexual behavior in temporal lobe epilepsy. A study of the effects of temporal lobectomy on sexual behavior. Arch Neurol 16:37-43
63. Hamed S, Mohamed K, El-Taher A, Hamed E, Omar $H(2006)$ The sexual and reproductive health in men with generalized epilepsy:a multidisciplinary evaluation. Int JImpot Res 18:287-295

64. Wong M, Moss R (1992) Long-term and short-term electrophysiological effects of estrogen on the synaptic properties of hippocampal CA1 neurons. J Neurosci 12:3217-3225

65. Frye CA, Rhodes ME, Walf AA, Harney JP (2001) Testosterone reduces pentylenetetrazole-induced ictal activity of wild type mice but not those deficient in type I 5a-reductase. Brain Res 918:182-186 